\title{
The Implementation of Identity Based Routing Protocol in IOT Environment
}

\author{
IsraaAkramAlzuabidi \\ University of Baghdad/collage of arts/unit of computer \\ Prof dr.SeyedMortezaBabamir \\ University of kashn/Faculty Of Electrical and Computer Engineering
}

\begin{abstract}
Identity routing itself deals with the addresses that refers to only the identity of the host and not its location. Internet of things on the other hand is a concept that allows the connection of device via internet as well as communication of this devices with the owner. Information is transferred back and forth between devices and their respective owner. Singling out a specific device and establishing communication is on the basis of routing principles. The implementation of this concept especially in our everyday life would mean digitalizing ones home to better function more efficiently at work.
\end{abstract}

Keywords:Routing, Identity based, Internet of Things, Address, Topology, Communication, Connection, Information, Devices and Host.

DOI: $10.7176 / \mathrm{CEIS} / 10-2-03$

Publication date:March $31^{\text {st }} 2019$

\section{Overview}

Routing is done based on assigning addresses which basically depends on the host's location on the network. This is also dependent on the topology as well. One of the advantages of topology based addressing is that it improves scalability. Identity based routing deals with addresses that refer to only the identity of the host and not its location. Internet of Things (OIT) is not a new concept but took a long time for the implementation of it into modern day to day business activities as well as domestic routines. OIT allows devices to be connected over the internet and further enables them to communicate with each other. Talking to each other in this regards refers to the devices in constant communication with the owner.

For them to be able to communicate i.e. the owner and the device, information is sent from a source and received at a destination. For the owner to be able specifically communicate with a device in a distinct location also falls down on routing basics and principles which is essential as the path to send information before it can get to destination and further carry out the instruction it contains becomes the most important thing.

The working environment has become more demanding in the past couple of years which means more is expected from the working population. This means more time is spent at the office than one's home. In extreme weather conditions where it is very hot or very cold, it takes a while for the effect of the heater or Air conditioner to be felt when its turn on. Apart from that, a lot more other things that one needs to do on getting home has to wait until one is physically there to operate these devices.

If devices can be communicated with over the internet, then it means all devices in one's home can be connected and controlled remotely prior to ones arrival. This leads to point where a lot of questions begins to come up. Question such as what is Identity Based Routing Protocol (IBRP)? What does IBRP entails? What is Internet of Things (IOT)? How possible is it to implement IBP on IOT? What are the possible effects and impacts?

\section{Identity Based Routing Protocol (IBRP)}

It has been established that routing today is measured by the ability to assign addresses which is totally dependent on topological location in a given network. Although this leads to the improvement of scalability though topology based addressing, it doesn't change the fact that addresses may eventually be aggregated into blocks and further showcased as a single unit.

Another established fact is that the movement of host or network topology means the corresponding address much change automatically. The resulting problems are two: in ad-hoc network and sensornets and complications arising from the use of topology based addressing on the internet

Identity based protocol or addressing has been researched and discovered to be able to solve these issues although pose more severe problems towards scalability. Thisis because in identity based routing or addressing, the address only refers to the identity of the host but not its location

Characteristics of Identity Based Routing includes:

1. Usually enhances traditional access control system

2. It facilitates granular resource access

3. It further reduce attack surface 
4. It allows multiple entry point

5. It authenticates user dynamically and routes to appropriate end point

6. Safe and information leaked can be used to attack it

An overview is given

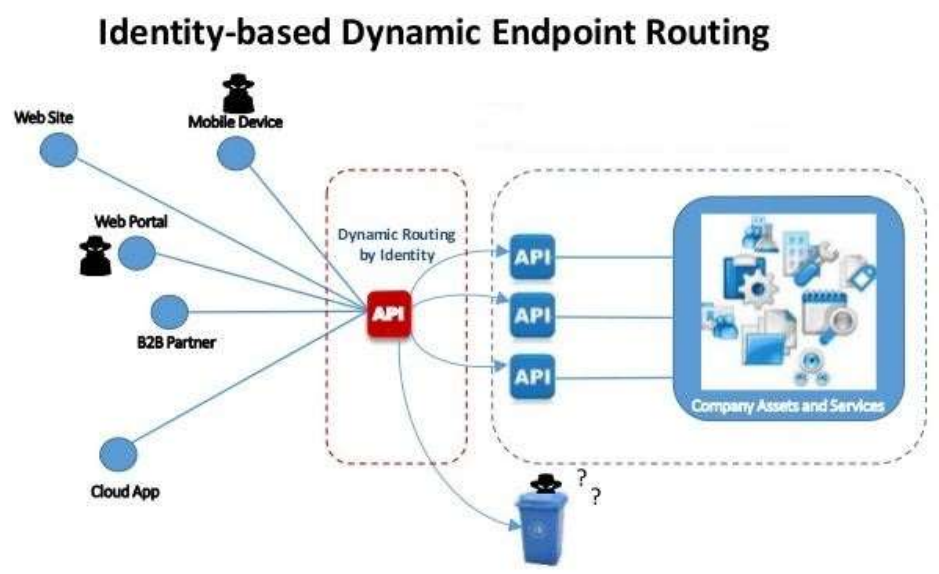

\section{Internet of Things (IOT)}

The internet of things is a phrase many are so familiar with but might not be sure if they really know what it means or not. Meola (2016) described it saying "The IoT refers to the connection of devices (other than typical fare such as computers and smartphones) to the Internet."

A more details and well explanatory definition was given by Rouse (2016) as "The Internet of Things (IoT) is a system of interrelated computing devices, mechanical and digital machines, objects, animals or people that are provided with unique identifiers and the ability to transfer data over a network without requiring human-to-human or human-to-computer interaction."

In short, Internet of Things involves data transfer over the network from a system of interrelated devices with unique identifiers. Understanding how the IOT works will help a lot in the event of an overview of the framework. The framework is very important as it will determine how identify based routing is integrated into it. Some peculiar terms must be used when discussing IOT in order to communicate a language universally understood by all. These terms are:

- Internet of Things: is described as a network of connected objects via internet that exchange data.

- Devices: this describes anything that can be control from a remote location via the internet. It can be appliances at home

- Entity: entity refers to businesses, consumers and even government that utilises internet of Things

- Physical layer: this is used to describe the hardware that makes IOT device gear.

- Network layer: this also describes the environment responsible for transmission of data that must have been collected in the physical layer to a different device

- Application layer: this accommodates all protocols as well as interfaces all devices use to first identify and then communicate with other devices

- Remotes: this is better explained as remote location where entities that utilise IOT devices connect and can be control from another location using a dashboard. Devices that can be controlled includes electrical appliances.

- Dashboard: this represents an interface or ecosystem where devices can be control

- Analytics: it very important to analyse data gathered or collected for any purpose and this represents the a system that analyses data generated by IOT devices

- Data storage: it simply represent location that acts as storage for data collected from IOT devices

- Networks: internet of things involves communication between a user and device or two devices over the internet

\section{Internet of Things (IOT) Framework}

The framework is described to have 5 main components which are user, dashboard, Internet connection, devices and data as all these interact in one way or the other to complete the cycle. The User has access to the dashboard which acts like a remote control to the devices. Communication occurs over the internet and data is passed from one end to the other. This is demonstrated below 


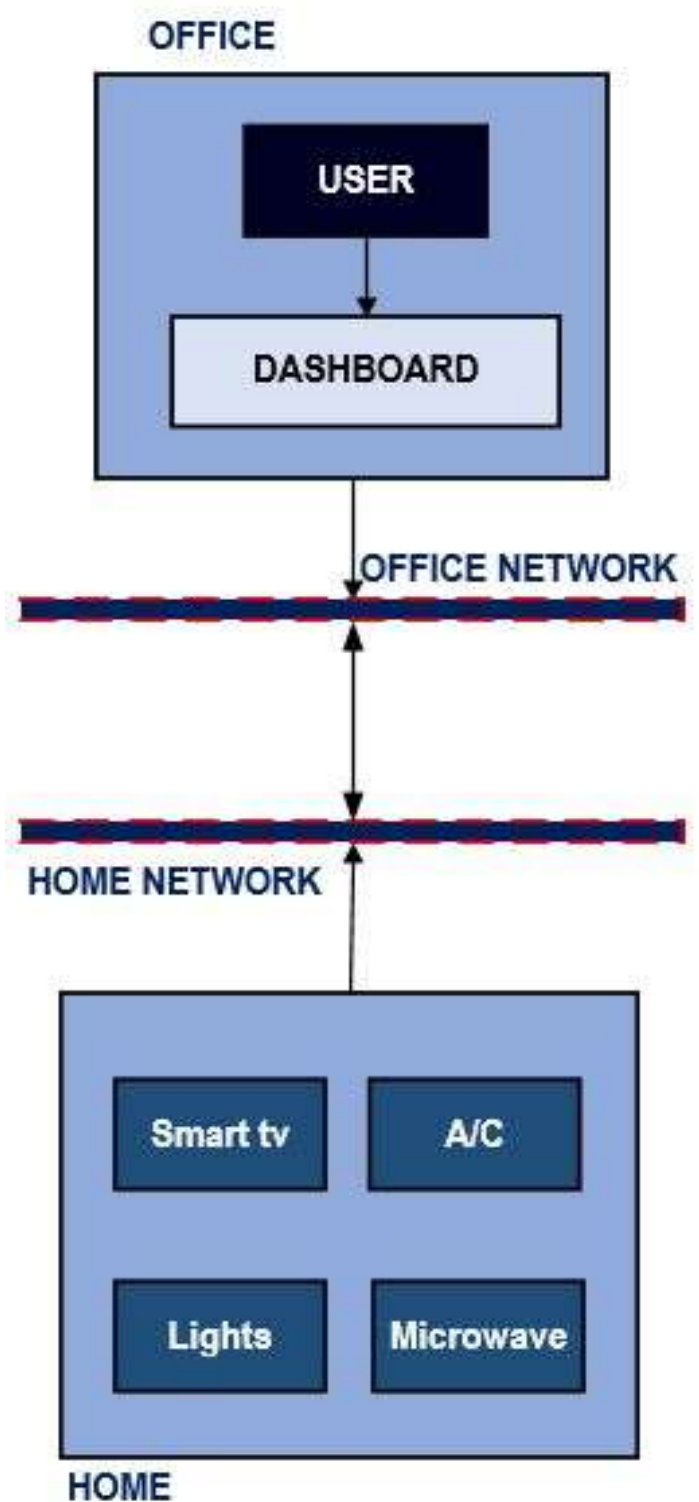

Implementing Identity Based Protocol on Internet of Things

As described earlier that "the movement of host or network topology means the corresponding address much change automatically". This creates two problems that Identity based protocol or addressing has been able to solve. The main reason for this is because "identity based routing or addressing, the address only refers to the identity of the host but not its location"'

This means location is not taken into consideration except the host, irrespective of the location, once the host is identified, routing follows that path. This happens over a network. The Internet of Things also allows communication over a network in order for a user to establish communication and pass instructions to devices. If the concept of Identity Based Routing is integrated into Internet of Things would only mean that user can target specific devices irrespective of location and communicate with them using dashboard or whatever tool is available to initiate instructions.

Just imagine parents remotely controlling the timing for kids to watch TV when they are not around or remotely controlling an electronic alarm to remind kids of their chores and assignments while away from home.

The reality of implementation of integrating Identity Based Routing and Internet of Things cannot be over emphasised as it gives way for a new trend of technological innovation and inventions which allows domestic and corporate devices to be control remotely.

The impact of the integration is felt more in executing more activities from one single location while the effect is clearly seen in the ease it brings along with the multi-process that would be completed within the shortest possible time. 
Effects and impact of implementation of identity based routing and internet of things.

There are numerous effects and impacts of the implementation of Identity based routing and Internet of Things which also depends on a different scenarios like industries and processes.

Production and Manufacturing Industry: In the production industry, large machinery that works for longer time can be control remotely by enabling them to be controlled over the internet. This makes production more efficient as there won't be any need for physical presence of an employee to physically switch on and off the machines.

Automobile Industry: The automobile industry is one characterised with a lot of automated processes and as such these numerous processes have to be monitored by personnel assigned to them. This is very similar to the production and manufacturing but this is more into automated systems monitoring

Security: It can also be integrated into securing homes and corporate places. This is done by integrating an alarm system into a home which can be controlled remotely. This can be used in corporate offices to secure building after closing. This can be activated when office hours are over and deactivate early enough each working day to enable employee gain access.

Education: In institutions were course that are practical oriented courses like engineering that has high power equipment, it can eb integrated to control access hours of students to the equipment and labs while they use it within restricted time frame so as to eb able to monitor it as well as guarantee the safety of these high power equipment.

In as much as one can go on with a lot of effects and impacts it can have, it is also important to critically evaluate types of application it can be integrated into in order to make regular and normal processes extremely easy.

\section{APPLICATIONS IMPLEMENTATION OF IDENTITY BASED ROUTING AND INTERNET OF THINGS}

There are quite a number of applications that the implementation of Identity Based Routing and Internet of Things is useful. The word "SMART" would be used consistently to mean "controlled from a remote location". These applications are

\section{Smart Homes}

In an instance where a studio apartment is rented by person who leaves the house in the morning and come home late in winter season means normally, he will get home first before coffee can be made, heater turned on and other domestic processes that requires a bit of time before been utilised. With the integration of IBR on IOT, it means he would have a situation where all these devices are connected to the internet and can issue commands to target specific device irrespective of his location.

The implication of this is that, he can decide to initiate coffee mixer to mix coffee ahead of his arrival or initiate the heater at home to start heating up the house before arriving at home or can simply initiate all the lights in the house to turn on to make it look as if there is actually someone at home.

\section{Smart Offices}

The implementation of IBR on IOT can also be integrated into corporate area to create a smart office. This becomes very handy especially in satiation where one is offering office space for rental or virtual office where the main entrance to the main building can be controlled remotely be enabling it to be connected to the internet. In this way, it creates a central opening and closing time for companies renting office space which everybody is expected to comply with. This also has effect on the security of the building as anyone found on the premises of the building during out of reach time is considered to be wandering and his actions are questionable

\section{Smart Labs}

Institutions are becoming more and electronic in natures and the needs to control a lot of processes and procedures have become important. This can be integrated into institutions in which lab doors, lights and even some basic equipment can be remotely controlled. Switched on when they need to be used and switched off when usage is done to avoid been left when not in use and been used when they are needed.

\section{Smart Malls}

In shopping malls everywhere, basic characteristics includes, numerous doors, escalators, high power air conditioners and security lights as well as cameras are all installed and meant to be used on a daily basis. Integrating IBP on IOT means all these can be controlled remotely without any issues whatsoever. Main doors can be opened as early as possible without physical presence of an employee. Escalators can also be controlled to work as early as possible unlike the tradition that escalators don't work until a certain time of the day.

Because it is a mall, it will be powered by high power air conditions and lights of which all can be remotely controlled. This is made so because each of these devices can be targeted and a command issued to them irrespective of the users location.

\section{Smart Stations}

In a city that has integrated computer controlled rail station, a central monitoring location can be built to allow control of all train stations access doors as well as the automated trains themselves. So as early as possible, from the central location, the train station's door can be controlled to open, and the trains can be controlled as well to 
move. All this can be controlled from one central location and the need for physical presence of staffs to open and close stations might not necessarily be needed.

\section{CONCLUSION}

The Identity based routing is a concept that can be well implemented to give rise to the way a lot of processes are now executed. This is also made possible with the help of Internet of Things which also has become a concept that people have started integrating into their respective daily lives because of the benefits that comes with it. Its effects and impact can be visualised in almost every industry which makes it considerable. Its applications are numerous and has the ability to create a lot more jobs worldwide and also creates new professions to match its rise.

\section{REFERENCES}

A. Lonergan (2016), Identity-based Endpoint Routing [Online]. Available on http://www.slideshare.net/AnthonyLonergan1/identitybased-endpoint-routing. Accessed on the $15^{\text {th }}$ of January, 2017

M. Rouse (2016), Internet of Things (IoT) [online]. Available on http://internetofthingsagenda.techtarget.com/definition/Internet-of-Things-IoT. Accessed on the $17^{\text {th }}$ of January, 2017

A. Meola (2016), what is the Internet of Things (IoT)? [Online]. Available on http://www.businessinsider.com/what-is-the-internet-of-things-definition-2016-8? IR=T\&r=US\&IR=T. Accessed on the $15^{\text {th }}$ of January, 2017

N. Kobie (2015), what is the internet of things? [Online]. Available on https://www.theguardian.com/technology/2015/may/06/what-is-the-internet-of-things-google. Accessed on the $19^{\text {th }}$ of January, 2017

M. Caesar, 2007, Identity-based routing [Online]. Available at http://digitalassets.lib.berkeley.edu/techreports/ucb/text/EECS-2007-114.pdf. Accessed on the 27 $7^{\text {th }}$ of September, 2016

B. Park, W. Lee, 2005, ISMANET: A Secure Routing Protocol using Identity-Based Signcryption Scheme for Mobile Ad-Hoc Networks [Online]. Available at https://ai2-s2pdfs.s3.amazonaws.com/ac26/30c06ae4f8f3026e55d9a7859ad9fbcf85d2.pdf. Accessed on the $26^{\text {th }}$ of September, 2016

R. Katz, 2007, Identity based Routing, [Online]. Available on http://dl.acm.org/citation.cfm?id=1467588. Accessed onthe $26^{\text {th }}$ of September, 2016

N. Kobie, 2015, what is the internet of things? [Online]. Available on https://www.theguardian.com/technology/2015/may/06/what-is-the-internet-of-things-google. Accessed on the $27^{\text {th }}$ of September, 2016

J. Morgan, 2014, A Simple Explanation Of 'The Internet Of Things [Online]. Available on http://www.forbes.com/sites/jacobmorgan/2014/05/13/simple-explanation-internet-things-that-anyone-canunderstand/\#4cc211b06828. Accessed on the $28^{\text {th }}$ of September, 2016

Sharma et al, 2009, Identity Based Secure Routing for Wireless Ad-Hoc Networks [Online]. Available on http://www.academypublisher.com/ijrte/vol02/no01/ijrte02012832.pdf. Accessed on the $28^{\text {th }}$ of September, 2016 G. Lu, S Jain, 2008, Virtual Id Routing: A scalable routing framework with support for mobility and routing efficiency [Online]. Available on http://conferences.sigcomm.org/sigcomm/2008/workshops/mobiarch/papers/p79.pdf. Accessed on the $28^{\text {th }}$ of September, 2016 